\title{
Evaluation of Cerebrospinal fluid adenosine deaminase activity as a complimentary tool in the early diagnosis of tuberculous meningitis in children
}

\author{
A. Pan, A. Biswas, A. Chaterjee, R. Kumar \\ Department of Pediatric Medicine, Medical College \& Hospital, Kolkata, College Street, Kolkata, India.
}

\begin{abstract}
The diagnosis of tuberculous meningitis cannot be made or excluded on the basis of clinical findings. Definite laboratory diagnosis is cumbersome and time consuming. Delay in diagnosis and institution of proper treatment is directly related to poor outcome and sequalae. Adenosine deaminase activity (ADA) was estimated in cerebrospinal fluid (CSF) in addition to routine CSF analysis of 32 patients of tuberculous meningitis (TBM) and 7 patients of partially treated pyomeningitis (PTM), 10 patients aseptic meningitis (AM) and 13 patients pyogenic meningitis (PM). Mean ADA levels in CSF of TBM patients were higher (15.42 U/L) as compared to 7.21 U/L, 6.41 U/L and 7.50 U/L in PTM, AM and PM respectively. This difference of ADA values in CSF between TBM and other types of meningitis was statistically significant $(p<0.01)$. ADA values were also compared with other biochemical and cytological parameters of CSF \& a positive correlation was found with CSF protein level. Sensitivity and specificity of ADA levels in CSF of children to diagnose tuberculous meningitis was $75 \%$ and $90 \%$ respectively at $10 \mathrm{U} / \mathrm{L}$ cut off of ADA levels in CSF.
\end{abstract}

\section{Key words: Tuberculous meningitis (TBM), adenosine deaminase activity(ADA), cerebrospinal fluid (CSF).}

\section{Introduction}

Tuberculous meningitis (TBM) still remains an important cause of morbidity and mortality in children. Often it poses a diagnostic problem to the clinicians and the prognosis of disease is closely related to the stage at which the treatment is started. The definitive diagnosis of TBM depends upon the detection of acid fast bacilli in the CSF and culture of Mycobacterium tuberculosis, which takes about 6-8 weeks. The

Correspondence: A. Pan

E-mail: atanu8k@yahoo.co.uk, chatanish123@indiatimes.com characteristic CSF cytological and biochemical changes also varies.

In India, nearly 5 lakhs people die due to tuberculosis each year. ${ }^{1}$ It represents $8.3 \%$ of childhood tuberculosis (TB). TBM complicates approximately 1 of every 300 untreated primary TB infections. The importance of this disease has increased since there is emergence of acquired immuno-deficiency syndrome (AIDS) and multidrug resistant TB. ${ }^{2}$ Delay in diagnosis and 
Journal of College of Medical Sciences-Nepal, 2012, Vol-8, No-2

institution of proper treatment is directly related to poor outcome and sequalae, which may be seen in 20-25 $\%$ of cases. ${ }^{3}$ In recent years, an elevated CSF adenosine deaminase (ADA) activity has been shown to be a promising test for the differentiation of patients of TBM from aseptic meningitis and controls but most of these studies are in adults. ${ }^{4}$ The results were less reliable in children. As a marker of cellular immunity, ADA activity is found to be elevated in those diseases in which there is a cell-mediated immune response. Raised levels of enzyme have been found in tuberculous pleural $^{5}$, peritoneal and pericardial fluids and cerebrospinal fluid (CSF) of patients with TBM. ${ }^{4,6}$ In view of these observations ADA activity was estimated in CSF of children with Tubeculous meningitis, Partially treated meningitis, Aseptic meningitis \& Pyogenic meningitis to assess its diagnostic value for TBM and to correlate with stages of TB and clinical parameters of CMI response to tuberculous infection.

\section{Place of Study}

Calcutta Medical College Hospital, Kolkata, West Bengal, India.

\section{Materials and methods}

This cross-sectional observational type of study was carried out from July 2007 to June 2008. All the patients in the age group 6 months to 12 years attending the pediatric inpatient ward during the above-mentioned time period, having the clinical or laboratory evidence of meningitis or meningoencephalitis were included in this study, thus a total 62 children were studied, of which there are 43 boys and 19 girls. All patients were divided in 4 groups as follows, with each group having their own inclusion criteria.

\section{Pyogenic meningitis (PM) group}

In this group, CSF of patients showing organisms in gram stained smear or culture or presence of bacterial antigen on latex agglutination was taken as diagnostic criteria. In the absence of organisms, CSF showed pleocytosis of more than 100 cells/ mm3 predominantly polymorphs, sugar less than half of blood sugar and protein more than $60 \mathrm{mg} \%$. This was taken as inclusion criteria.

\section{Partially treated pyomeningitis (PTM) group}

This group consisted of patients in whom their CSF showed presence of organisms on gram stained smear or culture or presence of bacterial antigen. In the absence of organism CSF showed pleocytosis of more than 100 cells $/ \mathrm{mm} 3$, sugar less than half of blood sugar, protein more than $60 \mathrm{mg} \%$ and who had received I.V. antibiotics for pyogenic meningitis for more than 48 hours before coming to hospital.

\section{Aseptic meningitis (AM) group}

This group consisted of patients whose CSF showed absence of organisms on gram stain or culture and CSF pleocytosis with more than 10 cells/mm3, predominantly lymphocytes and sugar more than $2 / 3$ of blood sugar value.

\section{Tuberculous meningitis (TBM) group}

In this group the patients had two or more of the following features on history; fever for $>$ two weeks, contact with an adult with tuberculosis, positive Mantoux test. This group had CSF with absolute lymphocyte counts $>50$ cells $/ \mathrm{mm} 3$, protein more than $60 \mathrm{mg} \%$ and sugar less than $2 / 3$ of blood sugar, chest $\mathrm{X}$-ray showing skiagram suggestive of pulmonary TB, 
A. Pan. Evaluation of Cerebrospinal fluid adenosine deaminase activity.

isolation of AFB from any site, CT scan showing evidence of chronic meningitis like hydrocephalus, basal exudates, infarcts, tuberculomas and histological evidence of tuberculosis.

No alterations were made in the treatment given to the child while at hospital. The standard regimen as suggested by consensus statement of Indian Academy of Pediatrics working group is followed at our hospital for treatment of TBM.

ADA activity in CSF was determined at $37^{\circ} \mathrm{C}$ according to the method of Guisti and Galanti ${ }^{7}$, based on the Berthlot reaction, that is the formation of colored indophenol complex from ammonia liberated from adenosine and quantified spectrphotometrically (U.V.Visible spectrophotometer. Remi. Model C-24). One unit of ADA is defined as the amount of enzyme required to release $1 \mathrm{mmol}$ of ammonia/min from adenosine at standard assay conditions. Results were expressed as units per litre per minute (U/L/min).

\section{Statistical analysis}

The means \pm standard error (SE) of ADA values was calculated for each group of subjects i.e. TBM, PM, PTM and AM and comparison was done among them, as shown in results. Vide Table.1

Test 1: TBM Vs. PTM : *p-value $<0.01(* *<0.01)$ Test 2: TBMVs. PM : *p-value $<0.01(* *<0.01)$ Test 3: TBM Vs. AM :*p-value $<0.01(* *<0.01)$ *Test: Two-sample Student t-test (Parametric Test: assuming normality)

**Test: Wilcoxon Two Sample Test (Nonparametric test: Without assuming any parametric distribution, in particular, normality)
We have also compared the relationship of ADA levels in CSF with other CSF parameters in TBM patients i.e. cell count, protein and sugar level. (Table. 3).

We consider tests at levels 0.01 and 0.1. p-value < 0.01 was taken as significant.

\section{Results and analysis}

We had a total of 62 subjects. Of them 43 were boys and 19 were girls. The mean age of children was 4.15 years in TBM, 3.90 years in PTM, 1.90 years in PM and 6.10 years in AM. Mean duration of illnesses was much higher in TBM group as compared with others. In TBM it is about 43days, whereas it is 9, 15 and 7 days respectively for PM PTM AM groups. The PM group had the highest mean cell counts and TBM group had the highest mean protein values. Among all the 62 patients only one had positive gram stained smear in the PTM group which shows gm+ve cocci in cluster. $\mathrm{Zn}$ stain showed also AFB only in one occasion. CSF culture was positive in 2 out of 13 patients of PM group. One patient grew Coagulase positive Staph. aureus from blood as well as for CSF. The other patient grew E.coli from CSF only. CSF culture for AFB shows growth of Mycobacterium tuberculosis in one occasion only. TB PCR has not done in all cases due to financial constraints. TB-PCR was positive on 3 occasions in TBM group.

The mean level of ADA in CSF was higher $(p<0.01)$ in TBM group of patients as compared to other groups (Table 1).

Mean ADA levels in CSF were lower (2.80 U/L) in case of pyogenic meningitis with low CSF cell counts as compared to TBM (16.20 U/L) with similar counts of cells. Mean ADA levels in CSF were also higher in 
Journal of College of Medical Sciences-Nepal, 2012, Vol-8, No-2

TBM (16.20 U/L), when compared with PTM and AM group with similar low CSF cell count. (table2)

In TBM group, levels of ADA in CSF were also correlated with cytological, \& biochemical parameters but found no correlation except CSF protein level. (table3).

The sensitivity of ADA test in CSF at cut off of $10 \mathrm{U} /$ $\mathrm{L}$ was $75 \%$. The specificity was highest while taking AM group as control (100\%) and was lowest while taking PM group as control (84.61\%). The overall specificity was $90 \%$.(table 4 )

At higher cut off of ADA value in CSF (11 U/L), the sensitivity decreases to $65.6 \%$ from $75 \%$. However, there was no change in the specificity. At lower cut off of ADA value in CSF (9U/L), sensitivity measured to $84 \%$ but specificity decreases from $90 \%$ to $83.3 \%$. So ADA levels of $10 \mathrm{U} / \mathrm{L}$ was taken as cut off value for diagnosis of TBM with overall sensitivity and specificity $75 \%$ and $90 \%$ respectively.(table 5 )

Table-1: ADA levels in CSF of four groups

\begin{tabular}{lcccc}
\hline A(U/L) & \multicolumn{3}{c}{} \\
\hline Groups & Mean & Median & Standard Error & Range \\
\hline TBM $(\mathrm{N}=32)$ & 15.42 & 13.80 & 0.95 & $7.60-28.90$ \\
PM $(\mathrm{n}=13)$ & 7.50 & 8.1 & 0.77 & $1.60-10.70$ \\
PTM $(\mathrm{n}=7)$ & 7.21 & 7.9 & 0.85 & $4.20-10.50$ \\
AM $(\mathrm{n}=10)$ & 6.41 & 7.2 & 1.16 & $1.10-10.60$ \\
\hline
\end{tabular}

Test 1: TBM Vs. PTM : *p-value $<0.01(* *<0.01)$

Test 2: TBM Vs. PM : *p-value $<0.01(* *<0.0 .1)$

Test 3: TBM Vs. AM : *p-value $<0.01(* *<0.01)$

Table-2: Variation of ADA levels in CSF with CSF pleocytosis group

\begin{tabular}{lcccc}
\hline \multicolumn{5}{c}{ Mean ADA Value (U/L) } \\
\hline $\operatorname{TBM}(\mathrm{n}=32)$ & 11.68 & 16.20 & 16.20 & 0 \\
$\operatorname{PM}(\mathrm{n}=13)$ & 0 & 0 & 2.8 & 7.99 \\
$\operatorname{PTM}(\mathrm{n}=7)$ & 0 & 4.2 & 8.1 & 6.96 \\
$\operatorname{AM}(\mathrm{n}=10)$ & 6.41 & 7.4 & 0 & 0 \\
& $<50$ & $50-100$ & $100-200$ & $>200$ \\
\hline Cell count (no. of cell/cmm) & & & \\
\hline
\end{tabular}


A. Pan. Evaluation of Cerebrospinal fluid adenosine deaminase activity.

Table-3: Relationship of ADA levels in CSF with other CSF parameters in TBM patients

\begin{tabular}{lcccc}
\hline & Mean & S.E. & Correlation & P-value \\
\hline ADA & & & Coefficient $(r)$ & \\
Total CSF Cell Count & 71.38 & 0.77 & - & - \\
Total CSF Protein & 207.37 & 14.89 & -0.10 & $\mathrm{p}>0.01(\mathrm{NS}) \mathrm{p}>0.1(\mathrm{NS})$ \\
Total CSF Sugar & 45.92 & 1.91 & -0.01 & $\mathrm{P}<0.01(\mathrm{~S})$ \\
\hline
\end{tabular}

* S: Significant **NS: Not Significant We consider tests at levels 0.01 and 0.1 .

Table-4: Sensitivity and specificity of the test at 10 U/L CSF ADA levels for diagnosis of TBM

\begin{tabular}{lcc}
\hline Groups & Sensitivity & Specificity \\
\hline TBM VS (PM+PTM+AM) & $75 \%$ & $90 \%$ \\
TBM VS PM & $75 \%$ & $84.61 \%$ \\
TBM VS PTM & $75 \%$ & $85.71 \%$ \\
TBM VS AM & $75 \%$ & $100 \%$ \\
\hline
\end{tabular}

Table-5: Sensitivity and specificity of ADA levels in CSF of patients at different ADA levels cut off

\begin{tabular}{ccc}
\hline TBM VS (PM+PTM+AM) & Sensitivity & specificity \\
\hline ADA cut off Values (U/L) & & \\
9 & $84 \%$ & $83.3 \%$ \\
10 & $75 \%$ & $90 \%$ \\
11 & $65.6 \%$ & $90 \%$ \\
\hline
\end{tabular}

\section{Discussion}

Results of our study indicate that ADA levels in CSF are of great value in diagnosis of TBM and in differentiating TBM from other meningitides i.e. PM, PTM \& AM.

Levels of ADA in CSF of adult patients of TBM have been evaluated in few earlier studies.

Raised levels of ADA in CSF are not specific to meningitis and this should be kept in mind when interpreting the test. Raised levels in other conditions particularly in certain intracrainal tumors has also been noted.
Previously conflicting results have been shown regarding the diagnostic value of CSFADA activity in children as compared to adult TBM patients.Many studies have demonstrated a high diagnostic value with sensitivity of $96-100$ per cent in adults. ${ }^{8,9}$

Ribera et. al. in their study had measured the activity of adenosine deaminase in cerebrospinal fluid for the diagnosis and follow-up of tuberculous meningitis in adults TBM patients, but they have not compared the ADA levels in CSF of TBM and PTM patients. ${ }^{4}$ 
Journal of College of Medical Sciences-Nepal, 2012, Vol-8, No-2

The mean ADA levels in CSF of TBM cases of pediatric age groups have been reported to be ranging between 11.6-13.7 U/L in earlier study. A relatively higher range of ADA values in CSF (15.7-21.3 U/L) has been observed in adult TBM patients. These results show that levels of ADA vary in different age groups and lower activity is observed in CSF of pediatric patients with TBM. This may be due to difference in immunological reactivity to tubercular antigen in children as compared to adults.

ADA assay has not been compared with PCR on pediatrics samples neither in the literature nor by us. We found a positive correlation of ADAlevels in CSF with CSF proteins but no significant correlation of ADA levels in CSF and CSF pleocytosis in our patients, whereas Malan et al. and Prasad et al found a positive correlation between CSF ADA levels, CSF proteins and CSF pleocytosis in patients of TBM. ${ }^{10},{ }^{11}$

None of the previous studies mentioned the ability of ADA levels in CSF to differentiate TBM from partially treated pyomeningitis.

Jakka S et al in a prospective cohort study, conducted on children admitted with a diagnosis of tuberculous meningitis. He compared levels in children with and without adverse neurological outcome. They have concluded that adverse neurological outcome in childhood tuberculous meningitis is associated with increased cerebrospinal fluid adenosine deaminase levels. In our study however we have not compared cerebrospinal fluid adenosine deaminase levels with final neurological outcome in childhood tuberculous meningitis. ${ }^{12}$
Our study was quite similar in terms of study design and result with the study by López-Cortés LF et al where CSF samples from 180 adults with meningitis of different etiologies were assayed for adenosine deaminase activity (ADA) and evaluated the usefulness of this assay in the differential diagnosis of aseptic meningitis. ${ }^{13}$

In conclusion, ADA estimation in CSF is a simple, inexpensive, rapid and fairly specific method for aiding a clinician in making the diagnosis of tuberculous meningitis when confronted with a common dilemma of distinguishing it from partially treated pyomeningitis and other meningitides. For above reasons, ADA estimation in CSF of TBM patients should find a place in routine laboratory methodology.

\section{References}

1. P.N. Tandon, Neurotuberculosis: Clinical aspects. In: Neurology in Tropics. Chopra, J.S. and Sawhney, I.M.S. (eds.) Churchill Livingstone Ltd.1999; 358-89.

2. G. Thweites, T.T.H., Chan, N.T.H. Ma et al. Tuberculous meningitis. J Neurol Neurosurg and Psychait 2000; 68: 289-99.

3. Garcia-Monco, J.C. CNS Tuberculosis. In: Neurologic Clinics. Marra, C.M. (ed.). 1999; 17(4):737-60.

4. E. Ribera, J.M. Martinez-Vazquez, I. Ocana, et al, Activity of adenosine deaminase in cerebrospinal fluid for the diagnosis and follow-up of tuberculous meningitis in adults. J Infect Dis 1987;155(4):603-7.

5. J.L. Banales, P.R. Pineda, J.M. Fitzgerald et al. Adenosine deaminase in the diagnosis of tuberculous pleura] effusions. A report of 218 patients and review of the literature Chest.1991;99:355-7. 
A. Pan. Evaluation of Cerebrospinal fluid adenosine deaminase activity...

6. M.A. Piras, C. Gatris. Cerebrospinal fluid adenosine deaminase activity in tuberculous meningitis. Enzyme. 1973; 14:311-17.

7. G. Giusti, Adenosine deaminase. In: Methods of enzyme analysis. Bergmeyer, H.U. (ed.). New York: Academic Press. 1974;1092-9.

8. T. Pettersson, M. Klockars, T.H. Weber. Diagnostic value of cerebrospinal fluid adenosine deaminase determination. Scand J Infect Dis. 1991; 23: 97-100.

9. R.M. Segura, C. Pascual, I. Ocana. et al. Adenosine deaminase in body fluids: a useful diagnostic tool in tuberculosis, d in Biochem. 1989; 22: 141-8.

10. C. Malan, P.R. Donald, M. Golden. et al Adenosine Deaminase levels in cerebrospinal fluid in the diagnosis of tuberculous meningitis. J. Trop. Med. Hy. 1984; 87: 33-40.

11. R. Prasad, A. Kumar, B.K. Khanna et al. Adenosine deaminase activity in cerebrospinal fluid for the diagnosis of tuberculous meningitis. Ind J Tub 1991;38: 99-102.

12. S. Jakka, S. Veena, A.R.Rao. et al Cerebrospinal fluid adenosine deaminase levels and adverse neurological outcome in pediatric tuberculous meningitis. : Infection. 2005;33(4):264-6.

13. L.F. López-Cortés, M. Cruz-Ruiz. et al. Adenosine deaminase activity in the CSF of patients with aseptic meningitis: utility in the diagnosis of tuberculous meningitis or neurobrucellosis. Clin Infect Dis. 1995; 20(3):525-30. 